\title{
Diagnostic and Treatment Approaches for Refractory Peptic Ulcers
}

\author{
Heung Up Kim \\ Department of Internal Medicine, Jeju National University School of Medicine, Jeju, Korea
}

Refractory peptic ulcers are defined as ulcers that do not heal completely after 8 to 12 weeks of standard anti-secretory drug treatment. The most common causes of refractory ulcers are persistent Helicobacter pylori infection and use of nonsteroidal anti-inflammatory drugs (NSAIDs). Simultaneous use of two or more H. pylori diagnostic methods are recommended for increased sensitivity. Serologic tests may be useful for patients currently taking proton pump inhibitors (PPIs) or for suspected false negative results, as they are not affected by PPI use. NSAID use should be discontinued when possible. Platelet cyclooxygenase activity tests can confirm surreptitious use of NSAIDs or aspirin. Cigarette smoking can delay ulcer healing. Therefore, patients who smoke should be encouraged to quit. Zollinger-Ellison syndrome (ZES) is a rare but important cause of refractory gastroduodenal ulcers. Fasting plasma gastrin levels should be checked if ZES is suspected. If an ulcer is refractory despite a full course of standard PPI treatment, the dose should be doubled and administration of another type of PPI considered. Clin Endosc 2015;48:285-290

Key Words: Peptic ulcer; Ulcer, refractory; Helicobacter pylori; Anti-inflammatory agent, non-steroidal; Gastrins

\section{INTRODUCTION}

Peptic ulcers were previously considered a chronic recurrent disease. However, a breakthrough in treatment of peptic ulcers followed the discovery of the causative role of Helicobacter pylori infection and introduction of proton pump inhibitors (PPIs) as powerful anti-secretory drugs.

However, because medical advances allow patients with serious diseases to survive longer and because an increasing number of older people use nonsteroidal anti-inflammatory drugs (NSAIDs), our hospital has experienced an increase in the number of peptic ulcer disease cases due to various causes.

PPI is the most powerful remedy for treatment of peptic ulcers. However, some peptic ulcers do not heal completely even with PPI treatment. Refractory ulcers are defined as peptic ul-

Received: April 17, 2015 Revised: May 20, 2015

Accepted: May 22, 2015

Correspondence: Heung Up Kim

Department of Internal Medicine, Jeju National University School of Medicine, 15 Aran 13-gil, Jeju 690-767, Korea

Tel: +82-64-717-1130, Fax: +82-64-717-1131, E-mail: kimhup@jejunu.ac.kr

(c) This is an Open Access article distributed under the terms of the Creative Commons Attribution Non-Commercial License (http://creativecommons.org/ licenses/by-nc/3.0) which permits unrestricted non-commercial use, distribution, and reproduction in any medium, provided the original work is properly cited. cers that do not heal completely despite 8 to 12 weeks of standard anti-secretory drug treatment. Patients with refractory peptic ulcers are generally believed to have persistent $H$. pylori infections or resistant strains, and these ulcers typically result from NSAID use, large size, malignancy, refractory response to drug administration, or other acid hypersecretory states. ${ }^{1}$ This article describes the diagnosis and treatment of refractory peptic ulcers.

\section{DIAGNOSIS AND APPROACH}

\section{Korean diagnostic guidelines}

Recent Korean guidelines for treatment of non-bleeding peptic ulcer disease recommend the following steps for treatment of refractory ulcers. ${ }^{2}$ The first is drug compliance. If an ulcer fails to heal with a standard dose of an anti-secretory drug, the dose should be doubled and treatment should be continued for an additional 6 to 8 weeks. Second, H. pylori status should be evaluated. False-negative results should be suspected when ulcers test negative for $H$. pylori infection. Third, clinicians should confirm that patients have discontinued use of NSAIDs. Many patients do not realize that cold remedies or headache medicines contain NSAIDs, so it is important to 
confirm that they are not inadvertently taking medicines containing NSAIDs. Fourth, clinicians should check that patients have quit smoking. Fifth, it is important to determine if there is a family history of gastrinoma, Zollinger-Ellison syndrome (ZES), or type I multiple endocrine neoplasia; their secondary symptoms, including chronic diarrhea or hypercalcemia due to hyperparathyroidism, should be investigated for acid hypersecretion. Sixth, the possibility of primary or metastatic malignancies, infectious diseases such as cytomegalovirus infection, crack cocaine use, and gastroduodenal involvement of inflammatory bowel disease should be considered. About $90 \%$ of refractory ulcers heal after 8 weeks of PPI treatment; however, additional or continuous treatment may be necessary in $10 \%$ of cases, and surgical treatment may also be considered.

\section{Clinical conditions}

\section{Persistent H. pylori infection}

Peptic ulcers were previously considered a chronic intractable disease because over $60 \%$ of ulcers recurred if their cause was not corrected. Several methods are used to detect H. pylori in the stomach, including culture, rapid urease tests, urea breath tests, histological examination, and stool antigen tests. However, these tests have pitfalls and introduce the potential for diagnostic errors. ${ }^{3}$ Recurrence rates have recently decreased with aggressive eradication of $H$. pylori-positive ulcers. However, false negative test results may result in overlooked $H$. pylori infections and missed standard treatment opportunities. Use of antibiotics, bismuth preparations, and PPIs reduce $H$. pylori numbers and can lead to false negative findings in many diagnostic modalities, including rapid urease, urea breath, and stool antigen tests, as well as culture and histological examination. Because PPIs decrease H. pylori numbers and also improve antral histology, an additional biopsy at the corpus is recommended after antral biopsy. In some cases, two or more simultaneous diagnostic methods are recommended. ${ }^{4}$ It is not difficult to determine $H$. pylori status during the first endoscopy. However, determination may become difficult after PPI treatment. In cases of failed eradication or false negative results, serologic tests may be used, as they are not affected by previous PPI use.

Non- $\mathrm{H}$. pylori helicobacter infections may also occur. $\mathrm{He}$ licobacter heilmannii has similar bacteriologic features, but is spread by zoonotic infection. ${ }^{5}$ The presenting symptoms of $H$. heilmannii infection are similar to those of $H$. pylori. Like $H$. pylori, H. heilmannii causes many diseases, including peptic ulcer diseases, gastric adenocarcinoma, and mucosa-associated lymphoid tissue lymphoma. Due to its low colonization density and the low sensitivity of the rapid urease tests, it is usually diagnosed by biopsy. Once diagnosed, the treatment is same as for H. pylori. ${ }^{5}$

\section{NSAIDs and ulcerogenic drugs}

NSAIDs delay ulcer healing by depleting prostaglandin, which plays a critical role in mucosal maintenance and protection. ${ }^{6}$ NSAID use is more frequently attributed to gastric ulcers, but may also result in duodenal ulcers. NSAIDs have recently emerged as a major cause of peptic ulcers in an increasingly aged population with increased NSAID use, as well as a decreased prevalence of $H$. pylori previously considered the primary cause of peptic ulcer diseases. There is a high possibility of abuse among patients using NSAIDs. ${ }^{7}$ The treatment of choice for ulcers due to NSAIDs use is discontinuation of NSAIDs. However, pain makes it difficult to stop NSAIDs use. Moreover, many patients are often unaware of their use of over-the-counter NSAIDs. There are some reports that the platelet cyclooxygenase (COX) activity test can confirm surreptitious use of NSAIDs or aspirin. ${ }^{1}$ The COX activity test reportedly revealed $21.5 \%$ more aspirin users than history alone. ${ }^{8}$ Selective COX-2 inhibitors have been developed to reduce adverse gastroduodenal effects of NSAIDs. However, delayed healing gastric ulcers in patients taking COX-2 inhibitors was comparable to that of patients taking ordinary NSAIDs. ${ }^{6}$ COX-2 inhibitor use is also limited due to risk of cardiovascular complications as well as decreased or complete elimination of selectivity when co-administered with aspirin. ${ }^{9}$

In addition to NSAIDs, many other drugs, including potassium chloride, bisphosphonates, ${ }^{10}$ clopidogrel, anti-cancer chemotherapeutic agents, and mycophenolate mofetil can cause peptic ulcers.

\section{Smoking}

Smoking may be an important factor in idiopathic refractory ulcers. ${ }^{11}$ It is one of the most important causes of refractory ulcers, and is listed in the current Korean guidelines. ${ }^{2}$ Smoking reduces prostaglandin synthesis and decreases the barrier function of the gastric mucosa. ${ }^{12}$ Cigarette smoking also induces a significant reduction in gastric mucosal blood flow. ${ }^{13}$ Peptic ulcers are more common in smokers than in non-smokers due to increased gastrin and gastric acid levels and decreased bicarbonate secretion. Cigarette smoking is positively associated with peptic ulcer pathogenesis and delayed ulcer healing. Several studies have shown that cigarette smoke and its active ingredients can cause mucosal cell death, inhibit cell renewal, decrease blood flow in the gastrointestinal mucosa, and interfere with the mucosal immune system. ${ }^{14,15}$ Therefore, patients who smoke should be encouraged to quit because continued smoking delays healing of peptic ulcers. ${ }^{16,17}$ 


\section{Crack cocaine}

The inhalable free-base form of cocaine is manufactured by processing the cocaine with ammonia or sodium bicarbonate (baking soda) to remove hydrochloride. ${ }^{18}$ This form is heat-stable and melts at $98^{\circ} \mathrm{C}$, which allows it to be smoked. ${ }^{18}$ Cocaine blocks presynaptic reuptake of norepinephrine and dopamine, and acts as powerful sympathomimetic agent. It also causes increased endothelin production and decreased nitric oxide production. ${ }^{18-20}$ Smoking crack cocaine induces tissue ischemia via contraction of mucosal arterioles. This process is repeated each time smoking occurs, leading to serious stomach and intestinal ulcers. ${ }^{21}$ Though it is typically difficult to obtain an accurate history from patients who smoke crack cocaine, this cause should be considered in cases of idiopathic refractory ulcers.

\section{Zollinger-Ellison syndrome}

ZES is defined as the presence of a gastrin-secreting neuroendocrine tumor that results in hypersecretion of gastric acid; ${ }^{22}$ it commonly manifests as peptic ulcer disease, dyspepsia, gastroesophageal reflux, abdominal pain, and diarrhea. ${ }^{23}$ The hallmark of this condition is hypergastrinemia in the presence of low gastric $\mathrm{pH}^{24}$

ZES is a rare disease, but is one of the most important causes of refractory gastroduodenal ulcers. The ulcers are not completely healed with standard anti-secretory agent therapy and rapidly recur after treatment cessation. Diarrhea may be due to acid hypersecretion and can be improved with use of anti-secretory agents. While the gastric mucosa is relatively normal, the gastric folds are hypertrophied. This syndrome is characterized by multiple ulcers beyond the duodenal bulb. Due to the gastrin-secreting tumor, ${ }^{25}$ fasting plasma gastrin concentrations greater than $1,000 \mathrm{pg} / \mathrm{mL}$ or basal acid outputs (BAOs) greater than $15 \mathrm{mEq} / \mathrm{hr}$ suggest the presence of ZES. Because use of anti-secretory drugs increases blood gastrin levels, these drugs should be withdrawn before measurement. However, it may be difficult to stop anti-secretory agents in cases of refractory ulcers. The most common causes of gastrin elevation are ZES and atrophic gastritis with hypochlorhydria. Moderate gastrin increases over 400 pg/mL make it difficult to diagnose ZES because this level is also seen in atrophic gastritis with hypochlorhydria, in which the gastrin levels increase due to a negative feedback mechanism by the hypochlorhydria. In this case, gastric acid secretion should be checked. If ZES is suspected despite moderate gastrin increase, secretin tests may be helpful. ${ }^{26}$ Clinical features may also help differentiate these conditions: acid hypersecretion, gastric wall hypertrophy, multiple duodenal ulcers, and increasing numbers of parietal cells are typical feature of ZES, while hypochlorhydria, gastric mucosal atrophy, and decreased numbers of parietal cells on histological examination characterize atrophic gastritis.

The secretin stimulation test is the best way to diagnose gastrinoma. Blood draws to measure serum gastrin levels should be obtained immediately before intravenous secretin administration and then $1,2,5,10,15$, and 30 minutes later. ZES is diagnosed based on serum gastrin concentrations increasing to $\geq 120 \mathrm{pg} / \mathrm{mL}^{27}$ The gastric acid output test is very complex; however, $\mathrm{pH}>2$ is inconsistent with $\mathrm{ZES}$.

Patients diagnosed with ZES must be tested to determine if the tumor is a type I multiple endocrine neoplasia. ${ }^{28}$ Somatostatin receptor scintigraphy is useful to localize the tumor site in about $80 \%$ of cases; endoscopic ultrasound also offers high sensitivity and specificity to accurately identify the tumor site.

Gastrinoma is treated by suppressing acid hypersecretion and tumor growth. ${ }^{29}$ Gastrinoma resection is the treatment of choice. PPIs are the best treatment for acid hypersecretion in gastrinoma, and should be used in doses sufficient to suppress BAO below $10 \mathrm{mEq} / \mathrm{hr}^{25}$

\section{Other acid hypersecretion states}

After ruling out $H$. pylori infection and ZES, idiopathic gastric acid hypersecretion should be considered for refractory peptic ulcers with acid hypersecretion. The diagnostic criteria are as follows: an increase in $\mathrm{BAO}$ over $10 \mathrm{mEq} / \mathrm{hr}$ with a fasting serum gastrin lower than $100 \mathrm{pg} / \mathrm{mL}$ or negative secretin test in the case of gastrin over $100 \mathrm{pg} / \mathrm{mL}^{30}$ Idiopathic gastric acid hypersecretion can be controlled with strong anti-secretory drugs. ${ }^{31}$

Gastric surgery can result in gastrin-induced acid hypersecretion. After partial gastrectomy with Billroth II anastomosis, a small portion of the antral mucosa containing G-cells may remain in the proximal portion of the duodenum. The gastric antral mucosa at the end of duodenal loop is stimulated by the alkaline duodenal environment to continuously secrete gastrin, a condition known as retained gastric antrum syndrome. This condition may be suspected if peptic ulcers recur after partial gastrectomy with Billroth II anastomosis, and can be differentiated by moderately increased serum gastrin levels and a more prominent increase in BAO over the peak acid output. $^{32}$

\section{Giant ulcers}

Giant ulcers are defined as gastric ulcers over $3 \mathrm{~cm}$ or duodenal ulcers over $2 \mathrm{~cm}^{33}$ Because giant ulcers require more time to heal, they typically fulfill the refractory ulcer criteria. In general, larger ulcers require more time to heal than smaller ulcers. ${ }^{34}$ Large ulcers are more common in old age, again requiring longer healing times under the same treatment con- 
ditions; about $13 \%$ of these ulcers meet the diagnostic criteria for giant ulcers. ${ }^{35}$

\section{Systemic disease such as Crohn disease and vasculitides}

The incidence of Crohn disease has recently increased so much in Korea that it is now a differential diagnosis of refractory ulcers. About $0.3 \%$ to $5 \%$ of Crohn disease cases involve the upper gastrointestinal tract. ${ }^{36}$ Cobblestone appearance, patch hyperemia or multiple erythema, mucosal friability, and protruding hypertrophy may be seen during endoscopic procedures, along with variable ulcer sizes. ${ }^{37}$ Though histological abnormalities may be visible on the duodenum and gastric antrum, the granuloma required for confirmation of Crohn disease is only seen in about 7\% of cases. ${ }^{21,38}$ About $16 \%$ of Crohn disease cases have abnormal endoscopy findings; however, only $7 \%$ have gastrointestinal symptoms. ${ }^{39}$

Refractory ulcers are also seen in various vasculitides such as polyarteritis nodosa, ${ }^{40}$ Henoch-Schönlein purpura, ${ }^{41}$ and eosinophilic gastroenteritis. ${ }^{42}$

\section{Mesenteric ischemia}

Blood supply to the stomach and duodenum is important to maintain mucosal integrity and promote ulcer healing. However, it is easy to overlook ischemia as a cause of peptic ulcers. Chronic mesenteric ischemia is an unusual cause of peptic ulcer disease because the gastroduodenal mucosa is supplied by rich vascularization. ${ }^{43}$ As the disease is typically asymptomatic until at least two major vessels are involved, it generally manifests only in individuals with a history of severe atherosclerotic disease. ${ }^{44}$ The typical symptoms are severe postprandial abdominal pain within 1 hour of ingestion, which subsides 1 to 2 hours later. Patients often limit food intake due to postprandial pain, resulting in substantial body weight loss. ${ }^{45}$ The goal of treatment is restoration of blood flow to the gastroduodenal mucosa, typically achieved with a vascular stent ${ }^{43}$ or operation.

\section{Infectious diseases}

Gastroduodenal infectious diseases such as tuberculosis, ${ }^{46}$ syphilis, ${ }^{47}$ strongyloidiasis ${ }^{48}$ cytomegalovirus or herpes virus infection, ${ }^{49}$ and mucormycosis ${ }^{50}$ can present as refractory ulcers. They should be diagnosed and treated with specific anti-microbial agents rather than with PPI.

\section{Chronic diseases}

Peptic ulcers are more common among patients with chronic diseases such as liver cirrhosis, chronic renal disease, and diabetes mellitus; these patients often have lower $H$. pylori eradication success rates and lower PPI efficacy than those without chronic diseases. ${ }^{51}$ It is, therefore, necessary to iden- tify underlying chronic diseases, if any, for cases of refractory ulcers.

\section{Radiation therapy}

The stomach and duodenum are sometimes involved in the radiation field during treatment of hepatocellular carcinoma, biliary-pancreatic cancer, or lymphoma. Radiation-induced ulcers are difficult to treat and usually fail to heal with conventional anti-acid secretory agents; they may require surgical treatment..$^{52}$ Both extracorporeal radiation and transarterial chemoembolization using radioisotopes can cause refractory ulcers. ${ }^{53}$

\section{Malignancy}

Endoscopies to screen for gastric cancer are popular in Korea. The aim of this procedure is to identify early cancers that typically present as ulcers. If the initial histological examination for an ulcer fails to diagnose a neoplastic lesion, clinicians concentrate on ulcer treatment. However, in the case of refractory ulcers, biopsies should be repeated if it is difficult to determine if the lesion is cancerous based on gross findings, even if the lesion suggests benign disease. Malignant ulcers may improve transiently with anti-secretory drug treatment, but aggravate with tumor progression. Follow-up endoscopy and repeated biopsy are recommended for benign-appearing ulcers.

\section{Treatment of refractory ulcers}

\section{Correction of the causes}

Before identification of their cause, peptic ulcers were considered a chronic recurrent disease. Without addressing these causes, healing might be delayed or ulcers could recur. Two or more H. pylori diagnostic methods may offer improved sensitivity. False negative findings should be suspected for cases of H. pylori-negative ulcers. If it is difficult to withhold PPI, physicians should consider serologic tests for $H$. pylori. NSAIDs should be stopped or changed to COX-2 inhibitors and physicians should survey for use of over-the-counter NSAIDs or NSAID patches. Patients should also refrain from smoking, and physicians should be aware of the signs of acid hypersecretion and the possibility of ZES. If the ulcer cause remains undetermined after following the above procedure, physicians should investigate for possible rare diseases.

\section{Anti-secretory drugs}

Anti-secretory drugs are used to improve the current state of ulcers, regardless of their cause. While various medications can be used to treat ulcers, PPIs are the most powerful. If an ulcer is refractory even after a full course of standard PPI 
therapy, the dose should be doubled and administration of another type of PPI should be considered.

\section{Surgical treatment}

Surgical treatment for refractory ulcers has markedly decreased in the PPI era; however, surgery is still necessary on a case-by-case basis. ${ }^{28}$ If it is not an emergency, surgery should be selected carefully based on consideration of patient compliance and willingness to change behavior.

\section{CONCLUSIONS}

The most common causes of refractory ulcers are $H$. pylori infection and chronic NSAID use; however, these factors are also the most common causes of peptic ulcers. Obtaining thorough patient histories and increasing efforts to identify hidden causes are necessary. Histological examination is important for final diagnosis of malignancy and identification of rare diseases such as infection or vasculitides. Therefore, histological examination of biopsy specimens taken during endoscopy is recommended. Finally, patients should be followed-up with a degree of skepticism in order to determine the underlying causes of refractory ulcers.

\section{Conflicts of Interest}

The author has no financial conflicts of interest.

\section{REFERENCES}

1. Lanas AI, Remacha B, Esteva F, Sáinz R. Risk factors associated with refractory peptic ulcers. Gastroenterology 1995;109:1124-1133.

2. Cheung DY, Jung HY, Song HJ, et al. Guidelines of treatment for non-bleeding peptic ulcer disease. Korean J Gastroenterol 2009;54:285297

3. Allahverdiyev AM, Bagirova M, Caliskan R, et al. Isolation and diagnosis of Helicobacter pylori by a new method: microcapillary culture. World J Gastroenterol 2015;21:2622-2628.

4. Choi YJ, Kim N, Lim J, et al. Accuracy of diagnostic tests for Helicobacter pylori in patients with peptic ulcer bleeding. Helicobacter 2012;17:77-85.

5. Bento-Miranda M, Figueiredo C. Helicobacter heilmannii sensu lato: an overview of the infection in humans. World J Gastroenterol 2014;20:17779-17787.

6. Schmassmann A. Mechanisms of ulcer healing and effects of nonsteroidal anti-inflammatory drugs. Am J Med 1998;104(3A):43S-51S.

7. Lanas A, Remacha B, Sáinz R, Hirschowitz BI. Study of outcome after targeted intervention for peptic ulcer resistant to acid suppression therapy. Am J Gastroenterol 2000;95:513-519.

8. Lanas A, Sekar MC, Hirschowitz BI. Objective evidence of aspirin use in both ulcer and nonulcer upper and lower gastrointestinal bleeding. Gastroenterology 1992;103:862-869.

9. Jones R, Rubin G, Berenbaum F, Scheiman J. Gastrointestinal and cardiovascular risks of nonsteroidal anti-inflammatory drugs. Am J Med 2008;121:464-474.

10. Graham DY. What the gastroenterologist should know about the gastro- intestinal safety profiles of bisphosphonates. Dig Dis Sci 2002;47:16651678.

11. Lanas A. NSAID use and abuse in gastroenterology: refractory peptic ulcers. Acta Gastroenterol Belg 1999;62:418-420.

12. Quimby GF, Bonnice CA, Burstein SH, Eastwood GL. Active smoking depresses prostaglandin synthesis in human gastric mucosa. Ann Intern Med 1986;104:616-619.

13. Iwao T, Toyonaga A, Ikegami M, et al. Gastric mucosal blood flow after smoking in healthy human beings assessed by laser Doppler flowmetry. Gastrointest Endosc 1993;39:400-403.

14. Li LF, Chan RL, Lu L, et al. Cigarette smoking and gastrointestinal diseases: the causal relationship and underlying molecular mechanisms (review). Int J Mol Med 2014;34:372-380.

15. Rantanen T, Udd M, Honkanen T, et al. Effect of omeprazole dose, nonsteroidal anti-inflammatory agents, and smoking on repair mechanisms in acute peptic ulcer bleeding. Dig Dis Sci 2014;59:2666-2674.

16. Doll R, Jones FA, Pygott F. Effect of smoking on the production and maintenance of gastric and duodenal ulcers. Lancet 1958;1:657-662.

17. Sonnenberg A, Müller-Lissner SA, Vogel E, et al. Predictors of duodenal ulcer healing and relapse. Gastroenterology 1981;81:1061-1067.

18. Lange RA, Hillis LD. Cardiovascular complications of cocaine use. N Engl J Med 2001;345:351-358.

19. Mo W, Singh AK, Arruda JA, Dunea G. Role of nitric oxide in cocaine-induced acute hypertension. Am J Hypertens 1998;11(6 Pt 1):708714.

20. Wilbert-Lampen U, Seliger C, Zilker T, Arendt RM. Cocaine increases the endothelial release of immunoreactive endothelin and its concentrations in human plasma and urine: reversal by coincubation with sigma-receptor antagonists. Circulation 1998;98:385-390.

21. Fennell DA, Gandhi SS, Prichard BN. Gastrointestinal haemorrhage associated with free-base (crack) cocaine. Postgrad Med J 1995;71:377-378.

22. Zollinger RM, Ellison EH. Primary peptic ulcerations of the jejunum associated with islet cell tumors of the pancreas. Ann Surg 1955;142:709723.

23. Jensen RT, Gardner JD, Raufman JP, Pandol SJ, Doppman JL, Collen MJ. Zollinger-Ellison syndrome: current concepts and management. Ann Intern Med 1983;98:59-75.

24. Isenberg JI, Walsh JH, Grossman MI. Zollinger-Ellison syndrome. Gastroenterology 1973;65:140-165.

25. Nieto JM, Pisegna JR. The role of proton pump inhibitors in the treatment of Zollinger-Ellison syndrome. Expert Opin Pharmacother 2006;7:169-175.

26. Guzzo JL, Duncan M, Bass BL, Bochicchio GV, Napolitano LM. Severe and refractory peptic ulcer disease: the diagnostic dilemma: case report and comprehensive review. Dig Dis Sci 2005;50:1999-2008.

27. Berna MJ, Hoffmann KM, Long SH, Serrano J, Gibril F, Jensen RT. Serum gastrin in Zollinger-Ellison syndrome: II. Prospective study of gastrin provocative testing in 293 patients from the National Institutes of Health and comparison with 537 cases from the literature. evaluation of diagnostic criteria, proposal of new criteria, and correlations with clinical and tumoral features. Medicine (Baltimore) 2006;85:331-364.

28. Napolitano L. Refractory peptic ulcer disease. Gastroenterol Clin North Am 2009;38:267-288.

29. Hung PD, Schubert ML, Mihas AA. Zollinger-Ellison syndrome. Curr Treat Options Gastroenterol 2003;6:163-170.

30. Lewis JH. Idiopathic gastric acid hypersecretion: treatment implications for refractory acid/peptic disorders. Aliment Pharmacol Ther 1991;5 Suppl 1:15-24.

31. Collen MJ, Wirshup JF. Ranitidine therapy in patients with idiopathic gastric acid hypersecretion. A prospective study. Dig Dis Sci 1995;40:1687-1690.

32. Webster MW, Barnes EL, Stremple JF. Serum gastrin levels in the differential diagnosis of recurrent peptic ulceration due to retained gastric antrum. Am J Surg 1978;135:248-252.

33. ASGE Standards of Practice Committee, Banerjee S, Cash BD, et al. The 
role of endoscopy in the management of patients with peptic ulcer disease. Gastrointest Endosc 2010;71:663-668.

34. O’Laughlin JC, Silvoso GK, Ivey KJ. Resistance to medical therapy of gastric ulcers in rheumatic disease patients taking aspirin. A double-blind study with cimetidine and follow-up. Dig Dis Sci 1982;27:976980

35. Raju GS, Bardhan KD, Royston C, Beresford J. Giant gastric ulcer: its natural history and outcome in the H2RA era. Am J Gastroenterol 1999;94:3478-3486.

36. Tan WC, Allan RN. Diffuse jejunoileitis of Crohn's disease. Gut 1993;34:1374-1378

37. Tanaka M, Kimura K, Sakai H, Yoshida Y, Saito K. Long-term follow-up for minute gastroduodenal lesions in Crohn's disease. Gastrointest Endosc 1986;32:206-209.

38. van Hogezand RA, Witte AM, Veenendaal RA, Wagtmans MJ, Lamers CB. Proximal Crohn's disease: review of the clinicopathologic features and therapy. Inflamm Bowel Dis 2001;7:328-337.

39. Annunziata ML, Caviglia R, Papparella LG, Cicala M. Upper gastrointestinal involvement of Crohn's disease: a prospective study on the role of upper endoscopy in the diagnostic work-up. Dig Dis Sci 2012;57:1618-1623.

40. Narusako T, Ueyama H, Tsunetomi N, et al. Multiple hemorrhagic gastric ulcers due to polyarteritis nodosa. Intern Med 1997;36:657-660.

41. Uza N, Yazumi S, Tanabe K, et al. Henoch-Schonlein purpura in an elderly patient with unusual manifestation. Endoscopy 2007;39 Suppl 1:E35-E36.

42. Raithel M, Hahn M, Donhuijsen K, et al. Eosinophilic gastroenteritis with refractory ulcer disease and gastrointestinal bleeding as a rare manifestation of seronegative gastrointestinal food allergy. Nutr J 2014;13:93.

43. Charoenpong P, Methachittiphan N, Peeraphatdit T, Naksuk N. Refractory peptic ulcer disease: listen for the cause. Am J Med 2014;127:e5-e6.

44. Biolato M, Miele L, Gasbarrini G, Grieco A. Abdominal angina. Am J Med Sci 2009;338:389-395.

45. Silva JA, White CJ. Ischemic bowel syndromes. Prim Care 2013;40:153167.

46. Lin OS, Wu SS, Yeh KT, Soon MS. Isolated gastric tuberculosis of the cardia. J Gastroenterol Hepatol 1999;14:258-261.

47. Jo JM, Kim HU, Heo ST. A case of gastric syphilis diagnosed by PCR test: a case report. J Med Life Sci 2014;11:43-47.

48. Thompson BF, Fry LC, Wells CD, et al. The spectrum of GI strongyloidiasis: an endoscopic-pathologic study. Gastrointest Endosc 2004;59:906910.

49. Hori K, Fukuda Y, Tomita T, Kosaka T, Noda S. Cytomegalovirus-associated gastritis. Gastrointest Endosc 2004;59:692-693.

50. Winkler S, Susani S, Willinger B, et al. Gastric mucormycosis due to Rhizopus oryzae in a renal transplant recipient. J Clin Microbiol 1996;34:2585-2587.

51. Kim JH, Moon JS, Jee SR, et al. Guidelines of treatment for peptic ulcer disease in special conditions. Korean J Gastroenterol 2009;54:318-327.

52. Zeng C, Luo S, Lv N, Chen Y. Refractory peptic ulceration following radiation therapy in primary gastric lymphoma: a report of two cases. Oncol Lett 2015;9:63-66.

53. Yim SY, Kim JD, Jung JY, et al. Gastrectomy for the treatment of refractory gastric ulceration after radioembolization with $90 \mathrm{Y}$ microspheres. Clin Mol Hepatol 2014;20:300-305. 